$\lim _{n=\infty} \frac{\pi}{n} \sum_{\nu=0}^{[n / 2]-1}\left(\frac{1}{\sin \frac{2 \nu+1}{2 n} \pi}-\frac{1}{\frac{2 \nu+1}{2 n} \pi}\right)$

or

$$
=\int_{0}^{\pi / 2}\left(\frac{1}{\sin x}-\frac{1}{x}\right) d x=\log \frac{4}{\pi},
$$

$$
\frac{2}{n} \sum_{\nu=0}^{[n / 2]-1} \frac{1}{\sin \frac{2 \nu+1}{2 n} \pi}=\frac{4}{\pi} \sum_{\nu=0}^{[n / 2]-1} \frac{1}{2 \nu+1}+\frac{2}{\pi} \log \frac{4}{\pi}+o(1) .
$$

Using the familiar asymptotic formula

$$
\sum_{\nu=0}^{m} \frac{1}{2 \nu+1}=\frac{1}{2} \log m+\frac{1}{2} C+o(1)
$$

where $C$ is Euler's constant, we find

$$
\frac{1}{n} \sum_{\nu=0}^{n-1} \frac{1}{\sin \frac{2 \nu+1}{2 n} \pi}=\frac{2}{\pi}\left(\log n+C+\log \frac{2}{\pi}\right)+o(1) .
$$

Technical Staff,

OfFICE OF THE ChIEF OF ORdNaNCE

\title{
THE MINIMUM AREA BETWEEN A CURVE AND ITS CAUSTIC.
}

\author{
BY PROFESSOR PAUL R. RIDER.
}

(Read before the American Mathematical Society April 9, 1920.)

If rays from a given source of light are reflected by a curve, the envelope of the rays after reflection is called the caustic of the curve. It is an interesting problem to find the curve which connects two fixed points and which with its caustic and the rays reflected from the fixed points will enclose a minimum area. Euler* proposed and solved a similar problem

* Euler, Methodus inveniendi lineas curvas maximi minimive proprietate gaudentes or German translation in Ostwald's Klassiker der exakten Wissenschaften, no. 46. See also Todhunter, Researches in the calculus of variations, chapter 13. 
concerning the curve which with its evolute and its normals at the fixed points will enclose the least area. The curves that furnish a solution of Euler's problem are cycloids. The curves that afford a solution of the problem concerning a curve and its caustic are transcendental curves of a more complex type. Their parametric equations are obtained in $\S 1$. The determination of the arbitrary constants that occur in the solution is considered in $\S 2^{*}$. In $\S 3$ a problem is solved which includes the evolute problem and the caustic problem as special cases.

\$1. Solution of the Problem. We shall consider the case in which the source of light is at an infinite distance, and shall assume that the rays are parallel to the $y$-axis. Let the two fixed points be $P_{0}\left(x_{0}, y_{0}\right)$ and $P_{1}\left(x_{1}, y_{1}\right), x_{0} \neq x_{1}$. We assume that the curve $y=y(x)$ which joins these two points and which with its caustic and the reflected rays at $P_{0}$ and $P_{1}$ encloses the minimum area, is single-valued with respect to $x$, and is continuous and possesses continuous derivatives.

If $\tau=\arctan y^{\prime}$, the equation of the reflected ray from the point $(x, y(x))$ is readily found to be $\dagger$

or

$$
Y-y(x)=-\cot 2 \tau \cdot(X-x),
$$

$$
Y-y(x)=\frac{y^{\prime 2}(x)-1}{2 y^{\prime}(x)}(X-x),
$$

where $X, Y$ are the current coordinates on the reflected ray.

Since the caustic is the envelope of the reflected rays, its parametric equations are obtained in the usual way by differentiating equation (1) with respect to the parameter $x$, and combining the new equation with (1). The equations of the caustic turn out to be

$$
X=x-\frac{y^{\prime}(x)}{y^{\prime \prime}(x)}, \quad Y=y(x)+\frac{1-y^{\prime 2}(x)}{2 y^{\prime \prime}(x)} .
$$

The distance from the point $(x, y(x))$ on the curve to the corresponding point $X, Y$ on the caustic is

$$
L=\frac{1+y^{\prime 2}(x)}{2 y^{\prime \prime}(x)}
$$

* Sufficient conditions, etc., will be treated in a later paper.

$\dagger$ Throughout the paper accents denote differentiation with respect to $x$. 
and the differential of area between the curve and its caustic is

$$
d A=\frac{1}{2} L \cos \tau \cdot d s=\frac{1+y^{\prime 2}(x)}{4 y^{\prime \prime}(x)} d x,
$$

where $d s$ is the element of arc on the curve. Thus our problem reduces to that of minimizing the integral

$$
A=\frac{1}{4} \int_{x_{0}}^{x_{1}} \frac{1+{y^{\prime 2}}^{2}(x)}{y^{\prime \prime}(x)} d x .
$$

By the usual process of the calculus of variations* it is found that if the integral $A$ is to be a minimum, $y(x)$ must satisfy the differential equation

$$
\frac{2 y^{\prime}}{y^{\prime \prime}}+\frac{d}{d x} \frac{1+{y^{\prime 2}}^{2}}{y^{\prime \prime 2}}=\text { const. }=2 a .
$$

Moreover, if the direction of the curve $y=y(x)$ at $P_{0}$ and $P_{1}$ is not prescribed, then we must have $\left(1+{y^{\prime}}^{2}\right) / y^{\prime \prime}=0$ at these points, that is the curve must have cusps at these points, and furthermore the caustic must pass through the points.

If we substitute $\tan \tau$ for $y^{\prime}$, we can easily reduce equation (2) to the form

$$
-\frac{\cos ^{2} \tau \cdot \tau^{\prime \prime}}{\tau^{\prime 3}}=a,
$$

from which we find

$$
-\frac{\tau^{\prime \prime}}{\tau^{\prime 2}}=a \sec ^{2} \tau \cdot \tau^{\prime}
$$

Integrating this equation, we get

$$
\frac{1}{\tau^{\prime}}=a \tan \tau+b,
$$

or

This gives

$$
d x=a \tan \tau d \tau+b d \tau .
$$

$$
x=a \log \sec \tau+b \tau+c,
$$

or, if we set $p=y^{\prime}=\tan \tau$,

$$
x=a \log \sqrt{1+p^{2}}+b \arctan p+c .
$$

* See Bolza, Vorlesungen über Variationsrechnung, p. 152, Ex. 43. 
Differentiating (3) with respect to $y$, we get

$$
\frac{1}{p}=\frac{a p+b}{1+p^{2}} \frac{d p}{d y}
$$

from which we find that

$$
y=b \log \sqrt{1+p^{2}}-a \arctan p+a p+d .
$$

Thus the parametric equations of the minimizing curves are $\left\{\begin{array}{r}x-c=a \log \sqrt{1+p^{2}}+b \arctan p=a \log \sec \tau+b \tau, \\ y-d=b \log \sqrt{1+p^{2}}-a \arctan p+a p \\ =b \log \sec \tau-a \tau+a \tan \tau .\end{array}\right.$

The area between a curve of this set and its caustic is

$$
\begin{aligned}
A= & \frac{1}{4} \int_{x_{0}}^{x_{1}} \frac{1+{y^{\prime}}^{2}}{y^{\prime \prime}} d x=\frac{1}{4} \int_{p_{0}}^{p_{1}} \frac{(a p+b)^{2}}{1+p^{2}} d p \\
= & \frac{a^{2}}{4}\left(p_{1}-p_{0}\right)+\frac{a b}{2} \log \sqrt{\frac{1+p_{1}^{2}}{1+p_{0}^{2}}} \\
& +\frac{b^{2}-a^{2}}{4}\left(\arctan p_{1}-\arctan p_{0}\right) \\
=\frac{a^{2}}{4}\left(\tan \tau_{1}-\tan \tau_{0}\right)+ & \frac{a b}{2} \log \frac{\sec \tau_{1}}{\sec \tau_{0}}+\frac{b^{2}-a^{2}}{4}\left(\tau_{1}-\tau_{0}\right) .
\end{aligned}
$$

\$2. Determination of the Constants. If it is prescribed that the curve shall have the slope $p_{0}=\tan \tau_{0}$ at the point $P_{0}\left(x_{0}, y_{0}\right)$ and the slope $p_{1}=\tan \tau_{1}$ at the point $P_{1}\left(x_{1}, y_{1}\right)$, we have the following four equations from which to determine the constants $a, b, c, d$ :

(4) $\left\{\begin{array}{l}x_{i}=a \log \sqrt{1+p_{i}^{2}}+b \arctan p_{i}+c, \\ y_{i}=b \log \sqrt{1+p_{i}^{2}}-a \arctan p_{i}+a p_{i}+d, \quad(i=0,1) .\end{array}\right.$

The determinant of this system is

$$
\left|\begin{array}{llll}
\log \sqrt{1+p_{0}^{2}} & \arctan p_{0} & 1 & 0 \\
\log \sqrt{1+p_{1}^{2}} & \arctan p_{1} & 1 & 0 \\
p_{0}-\arctan p_{0} & \log \sqrt{1+p_{0}^{2}} & 0 & 1 \\
p_{1}-\arctan p_{1} & \log \sqrt{1+p_{1}^{2}} & 0 & 1
\end{array}\right|
$$


which reduces to

$\left(p_{1}-p_{0}\right)\left(\tau_{1}-\tau_{0}\right)-\left(\tau_{1}-\tau_{0}\right)^{2}$

Thus if

$$
-\left(\log \sqrt{1+p_{1}^{2}}-\log \sqrt{1+p_{0}^{2}}\right)^{2} .
$$

$$
\begin{aligned}
\left(p_{1}-p_{0}\right)\left(\tau_{1}-\tau_{0}\right) \neq\left(\tau_{1}-\tau_{0}\right)^{2} & \\
& +\left(\log \sqrt{1+p_{1}^{2}}-\log \sqrt{1+p_{0}^{2}}\right)^{2},
\end{aligned}
$$

the equations (4) can be solved for $a, b, c, d$.

§ 3. A more general Problem. If the integrand of the integral to be minimized is

$$
f=\left(1+p^{2}\right)^{m} q^{n}
$$

where $p=y^{\prime}, q=y^{\prime \prime}$, we have a type of problem which includes the caustic problem for $m=1, n=-1$, and Euler's evolute problem for $m=2, n=-1$.

It is found* that the minimizing curve must be a solution of the differential equation

$$
\frac{2 m(1-n) p}{1+p^{2}}-\frac{n(1-n) q^{\prime}}{q^{2}}=\frac{A}{\left(1+p^{2}\right)^{m} q^{n}},
$$

in which $A$ is an arbitrary constant. If we multiply both sides of equation (5) by $d p=q d x$, we get

$$
\frac{2 m(1-n) p}{1+p^{2}} d p+\frac{n(1-n)}{q} d q=\frac{A}{\left(1+p^{2}\right)^{m} q^{n}} d p,
$$

which reduces to

Integration gives

$$
(1-n) d \log f=\frac{A d p}{f} .
$$

$$
(1-n) f=A p+B .
$$

Replacing $f$ by $\left(1+p^{2}\right)^{m} q^{n}$ and solving for $q$ (that is $d p / d x$ ), we obtain

from which

$$
\frac{d p}{d x}=\frac{1}{(1-n)^{1 / n}} \frac{(A p+B)^{1 / n}}{\left(1+p^{2}\right)^{m / n}}
$$

$$
x=(1-n)^{1 / n} \int \frac{\left(1+p^{2}\right)^{m / n}}{(A p+B)^{1 / n}} d p .
$$

* See Bolza, loc. cit. 
Differentiating with respect to $y$, we get

$$
\frac{1}{p}=(1-n)^{1 / n} \frac{\left(1+p^{2}\right)^{m / n}}{(A p+B)^{1 / n}} \frac{d p}{d y},
$$

and consequently

$$
y=(1-n)^{1 / n} \int \frac{\left(1+p^{2}\right)^{m / n} p}{(A p+B)^{1 / n}} d p
$$

Thus (6) and (7) are the parametric equations of the minimizing curves.

WASHINGTON UNIVERSITY,

ST. Lours, Mo.

October 12, 1920.

\section{SHORTER NOTICES.}

Il Problema dei Tre Corpi da Newton ai Nostri Giorni. By R. Marcolongo. (Manuali Hoepli.) Milan, Ulrico Hoepli, 1919. $162 \mathrm{pp}$.

This little book, in the well known style of the Hoepli manuals, presents, as its title indicates, an account of the problem of three bodies from the time of Newton to the present. The author has limited himself strictly to a descriptive account of what has been accomplished in this interval of time, with full references to original memoirs and papers where the interested reader can find the complete developments.

Professor Marcolongo is well known as an authority in the field of dynamical systems, and this book from his pen will be welcomed by all who are interested in the development of mathematical astronomy. Here will be found references to the works of over 200 authors who have contributed to one or more phases of this celebrated problem, together with a short description of the aim, the method of attack, and the results attained.

The book is divided into six chapters. The title of each sufficiently indicates its content.

I. The works of the geometers of the eighteenth century.

II. Reduction of the differential equations to least order. 